\title{
TRANSITIVE SYSTEMS OF LINEAR OPERATORS ON A BANACH SPACE ${ }^{1}$
}

\section{BERTRAM YOOD}

Let $\mathfrak{X}$ be an infinite-dimensional Banach space. Mackey has shown, under more general conditions, that if $x_{1}, \cdots, x_{n}, y_{1}, \cdots, y_{n}$ are any two sets of $n$ linearly independent elements in $\mathfrak{X}$, then there exists an isomorphism $T$ of $\mathfrak{X}$ with itself such that $T\left(x_{i}\right)=y_{i}, i=1, \cdots, n$. In other words the collection of isomorphisms is transitive for linearly independent sets of elements of $\mathfrak{X}$. This property is shared by many other sets of linear operators, for example by the set of all operators with finite-dimensional range in $\mathfrak{X}$. These two sets of linear operators are semi-groups. ${ }^{3}$ In this note we give a condition for the transitivity property above which is necessary for all sets of linear operators and which while not in general sufficient is so for semi-groups. In this result the operators need not be assumed to be bounded or defined everywhere in $\mathfrak{X}$. $\mathfrak{E}(\mathfrak{X})$ will be used to designate the collection of all bounded linear operators with domain $\mathfrak{X}$ and range in $\mathfrak{X}$.

THEOREM 1. Let \& be a collection of linear operators with domain and range in an infinite-dimensional Banach space $\mathfrak{X}$. For \&5 to be transitive for linearly independent sets of elements of $\mathfrak{X}$ it is necessary that for each finite-dimensional subspace $F$ of $\mathfrak{X}$ there exists a $T \in \mathbb{B}$ which is the identity on $F$ and a number $\epsilon>0$ such that if $U \in \mathbb{E}(\mathfrak{X})$ takes $F$ into $F$ and $\|U\|<\epsilon$, then there exists an operator $V \in \circlearrowleft$ which agrees with $T+U$ on $F$. If Bs is a semi-group, this condition is sufficient.

It is readily seen that the condition is necessary. That it is not sufficient in general can be seen by taking ( $\$$ to be an $\epsilon$-neighborhood of the identity in the Banach space $\mathbb{E}(\mathfrak{X})$.

Let $\&$ be a semi-group. Let $F$ be a finite-dimensional subspace of $\mathfrak{X}$ and $T_{0}$ be its associated operator in $\mathcal{G}$, and let $x_{1}, \cdots, x_{n}$ be linearly independent elements of $F$. There exist $x_{i}{ }^{*}$ in $\mathfrak{X}^{*}$ (the conjugate space of $\mathfrak{X})$ such that $x_{i}^{*}\left(x_{j}\right)=\delta_{i j}, i, j=1, \cdots, n$. For elements

Received by the editors December 28, 1948 and, in revised form, June 13, 1949.

1 This paper is based on a portion of the author's dissertation, Yale University, 1947. The author wishes to thank the referee for his suggestions which made possible an appreciable shortening of proof of Theorem 1 and clarified the nature of that proof.

${ }^{2} \mathrm{G}$. W. Mackey, On infinite-dimensional linear spaces, Trans. Amer. Math. Soc. vol. 57 (1945) pp. 155-207, see Theorem II-3.

${ }^{3}$ By a semi-group of linear operators is meant a collection which contains $T U$ whenever it contains $T$ and $U$. 
$v_{i} \in F$ for which $\left\|v_{i}-x_{i}\right\|<\delta=\min \{1, \epsilon\} / 2 n \max \left\|x_{i}^{*}\right\|$, let the transformation $W$ be determined by

$$
W(x)=\sum_{i=1}^{n} x_{i}^{*}(x)\left(v_{i}-x_{i}\right) .
$$

Then $\|W\|<\min \{1, \epsilon\} / 2$. Thus $T_{0}+W$ is an isomorphism on $F$. The operator $U=\sum_{i=1}^{\infty}(-1)^{i} W^{i}$ has the properties $\|U\|<\epsilon$ and $\left(T_{0}+U\right)\left(T_{0}+W\right) x=x$ for all $x$ in $F$. By hypothesis there exist transformations $U_{1}$ and $W_{1}$ in (S) which agree with $T_{0}+U$ and $T_{0}+W$ respectively in $F$. Then by the above $W_{1}\left(x_{i}\right)=v_{i}$ and $U_{1}\left(v_{i}\right)=x_{i}$, $i=1, \cdots, n$. If also for each $i,\left\|v_{i}^{\prime}-x_{i}\right\|<\delta, v_{i}^{\prime} \in F$, we may define $W_{1}^{\prime} \in(B)$ as above so that $W_{1}^{\prime}\left(x_{i}\right)=v_{i}^{\prime}$. Then $W_{1}^{\prime} U_{1}\left(v_{i}\right)=v_{i}^{\prime}, i=1, \cdots$, $n$. The semi-group property of $\&$ shows that (a) for each $F$ and $x_{1}, \cdots, x_{n}$ linearly independent in $F$ there exists a $\delta>0$ such that for every $v_{i}, w_{i}$ in $F$ with $\left\|v_{i}-x_{i}\right\|<\delta$ and $\left\|w_{i}-x_{i}\right\|<\delta$, there exists $V$ in \& with $V\left(v_{i}\right)=w_{i}, i=1, \cdots, n$.

Next for a fixed $F$ and $n \leqq \operatorname{dim} F$ consider the Cartesian product space $\bar{F}=F \times \cdots \times F$ ( $n$ factors) made up of elements of the form $\bar{x}=\left(x_{1}, \cdots, x_{n}\right)$ with $\|\bar{x}\|=\max _{i \leqq n}\left\|x_{i}\right\| . \bar{F}$ is a finite-dimensional Banach space. ${ }^{4}$ Let $\bar{L}$ be the set of all $\bar{x} \in \bar{F}$ such that $x_{1}, \cdots, x_{n}$ are linearly independent in $F$. By (a), $\bar{L}$ is open in $\bar{F}$ and to each point $\bar{x}$ of $\bar{L}$ there exists a $\delta>0$ such that $\|\bar{x}-\bar{v}\|<\delta$ and $\|\bar{x}-\bar{v}\|<\delta$ imply the existence of a transformation $V \in(B)$ such that $\bar{V}(\bar{v})=\bar{w}$ where $\bar{V}(\bar{v})=\left(V\left(v_{1}\right), \cdots, V\left(v_{n}\right)\right)$. Let $\bar{K}$ be the set of $\bar{y}$ in $\bar{L}$ which can be reached from $\bar{x}$ along a finite chain of such spheres each overlapping its predecessor. Then by (a) for such a $\bar{y}$ there exists a sequence $V_{1}, \cdots, V_{r}$ in (S) such that for the product $W=V_{r} \cdots V_{1}, \bar{W}(\bar{x})=\bar{y}$ and by the semi-group property, $W \in \mathbb{S}$.

From this it follows by a standard topological argument, used in connection with analytic continuation and elsewhere, ${ }^{5}$ that the set $\bar{K}$ is open and closed in $\bar{L}$. Therefore (b) if $\bar{x}$ and $\bar{y}$ are in the same component of $\bar{L}$, there is a $V \in \mathbb{B}$ such that $\bar{V}(\bar{x})=\bar{y}$.

Call $\bar{x}$ and $\bar{y}$ fully independent if $x_{1}, \cdots, x_{n}, y_{1}, \cdots, y_{n}$ form a linearly independent set of $2 n$ elements and suppose that $\bar{x}, \bar{y} \in \bar{L}$. For such a pair $\bar{x}$ and $\bar{y}$, and for any real $\alpha$, we have $\alpha \bar{x}+(1-\alpha) \bar{y} \in \bar{L}$, for $\sum_{i=1}^{n} \beta_{i}\left(\alpha x_{i}+(1-\alpha) y_{i}\right)=0$ implies $\beta_{i}=0, i=1, \cdots, n$. Thus the line segment joining $\bar{x}$ and $\bar{y}$ is in $\bar{L}$. Therefore (c) if $\bar{x}$ and $\bar{y}$ are fully independent in $\bar{L}$, they must belong to the same component of $\bar{L}$.

${ }^{4} \mathrm{~S}$. Banach, Théorie des opérations linéaires, Warsaw, 1932, see p. 182.

${ }_{5}^{5}$ The use of the set $\bar{K}$ and of this argument was suggested by the referee to replace a more lengthy Heine-Borel argument. 
If $\bar{x}$ and $\bar{y}$ are two linearly independent sets of $n$ elements in $X$ which are not fully independent, consider a set $\bar{v}$ of $n$ elements which is fully independent of $\bar{x}$ and also of $\bar{y}$. Such a set $\bar{v}$ exists because $\mathfrak{X}$ is assumed to be infinite-dimensional. The subspace $F$ can be taken to contain all the vectors $x_{i}, y_{i}$, and $v_{i}$. For the $\bar{L}$ which corresponds to this $F, \bar{x}, \bar{y}$, and $\bar{v}$ must lie in the same component by (c). Then by (b) there exists a $U \in B($ with $\bar{U}(\bar{x})=\bar{y}$. This completes the proof.

Next we consider all our transformations as being in the space $\mathfrak{F}(\mathfrak{X})$. For $\mathfrak{E}(\mathfrak{X})$ the strong topology is defined, following the ideas of von Neumann, ${ }^{6}$ as that where the neighborhoods of a transformation $U_{0}$ are those of the form

$$
\begin{aligned}
& N\left(U_{0} ; x_{1}, \cdots, x_{k}, \epsilon\right) \\
& \quad=\left\{T \in \mathscr{E}(\mathfrak{X}) \mid\left\|T\left(x_{i}\right)-U_{0}\left(x_{i}\right)\right\|<\epsilon, i=1, \cdots, k\right\}
\end{aligned}
$$

where $\epsilon>0$ and $x_{i} \in \mathfrak{X}, i=1, \cdots, k$.

THEOREM 2. A subset $\mathfrak{A}$ of $\mathfrak{E}(\mathfrak{X})$ satisfying the conclusion of Theorem 1 is dense in the strong topology of $\mathbb{E}(\mathfrak{X})$.

It suffices to show that the neighborhood $N\left(U_{0} ; x_{1}, \cdots, x_{k}, \epsilon\right)$ contains an operator in $\mathfrak{A}$. By renumbering, if necessary, we let $x_{1}, \cdots, x_{r}$ be a linearly independent subset of the $x$ 's which generates the same linear manifold as is generated by all of them. We put $x_{i}=\sum_{j=1}^{r} a_{i j} x_{j}, i=r+1, \cdots, k$ and $A=\max \left|a_{i j}\right|$ for these values of $i$ and for $j=1, \cdots, r$. Also we set $\eta=\min \{\epsilon, \epsilon /(r A)\}$. We choose $w_{1}, \cdots, w_{r}$ in $\mathfrak{X}$ where each $w_{i}$ is linearly independent of the elements $U_{0}\left(x_{i}\right), i=1, \cdots, r$, and of the previously selected $w$ 's and each $\left\|w_{i}\right\|<\eta$. The collection $\left\{U_{0}\left(x_{i}\right)+w_{i}\right\}$ is a linearly independent set. By the assumption on $\mathfrak{A}$, there exists $T \in \mathfrak{A}$ such that $T\left(x_{i}\right)$ $=U_{0}\left(x_{i}\right)+w_{i}, i=1, \cdots, r$. Thus for these values of $i, \| T\left(x_{i}\right)$ $-U_{0}\left(x_{i}\right) \|<\epsilon$. For $i=r+1, \cdots, k$ we have

$$
\begin{aligned}
\left\|T\left(x_{i}\right)-U_{0}\left(x_{i}\right)\right\| & =\left\|\left(T-U_{0}\right)\left(\sum_{j=1}^{r} a_{i j} x_{j}\right)\right\| \\
& \leqq \sum_{j=1}^{r}\left|a_{i j}\right|\left\|T\left(x_{j}\right)-U_{0}\left(x_{j}\right)\right\|<\epsilon .
\end{aligned}
$$

Then $T \in N\left(U_{0} ; x_{1}, \cdots, x_{k} ; \epsilon\right)$.

Cornell University

${ }^{6} \mathrm{~J}$. von Neumann, Zur Algebra der Funktionaloperatoren und Theorie des normalen Operatoren. Math. Ann. vol. 102 (1929) pp. 370-427. 